Discrete Comput Geom 34:659-678 (2005)

DOI: $10.1007 / \mathrm{s} 00454-005-1203-1$

\title{
Quantitative Generalized Bertini-Sard Theorem for Smooth Affine Varieties*
}

\author{
Zbigniew Jelonek $^{1}$ and Krzysztof Kurdyka ${ }^{2}$ \\ ${ }^{1}$ Instytut Matematyczny, Polska Akademia Nauk, \\ Św. Tomasza 30, 31-027 Kraków, Poland \\ najelone@cyf-kr.edu.pl \\ ${ }^{2}$ Laboratoire de Mathématiques, Université de Savoie, CNRS UMR 5127, \\ Campus Scientifique, F-73376 Le Bourget du Lac cedex, France \\ kurdyka@univ-savoie.fr
}

To the memory of our teacher and friend Professor Stanislaw Lojasiewicz

\begin{abstract}
Let $X \subset \mathbb{C}^{n}$ be a smooth affine variety of dimension $n-r$ and let $f=$ $\left(f_{1}, \ldots, f_{m}\right): X \rightarrow \mathbb{C}^{m}$ be a polynomial dominant mapping. We prove that the set $K(f)$ of generalized critical values of $f$ (which always contains the bifurcation set $B(f)$ of $f$ ) is a proper algebraic subset of $\mathbb{C}^{m}$. We give an explicit upper bound for the degree of a hypersurface containing $K(f)$. If $I(X)$ - the ideal of $X$-is generated by polynomials of degree at most $D$ and $\operatorname{deg} f_{i} \leq d$, then $K(f)$ is contained in an algebraic hypersurface of degree at most $(d+(m-1)(d-1)+(D-1) r)^{n-r} D^{r}$. In particular if $X$ is a hypersurface of degree $D$ and $f: X \rightarrow \mathbb{C}$ is a polynomial of degree $d$, then $f$ has at most $(d+D-1)^{n-1} D$ generalized critical values. This bound is asymptotically optimal for $f$ linear. We give an algorithm to compute the set $K(f)$ effectively. Moreover, we obtain similar results in the real case.
\end{abstract}

\section{Introduction}

There is a quite abundant literature about singularities at infinity of polynomials $f: \mathbb{C}^{n} \rightarrow$ $\mathbb{C}$. This subject was initiated by $\mathrm{R}$. Thom who proved, some 30 years ago, that there is a finite set $B \in \mathbb{C}$ such that $f$ is a locally trivial fibration over the complement of $B$. The smallest such a set, denoted by $B(f)$, is called the set of atypical values of $f$. An effective (asymptotically sharp) bound for the number of points in $B(f)$ was given only

* The first author was partially supported by the MIRA Rhône-Alpes and by a Polish KBN grant. 
recently by the authors [10]. In this paper we propose a study of a much more general situation.

Let $X$ be a smooth affine variety over $k=\mathbb{R}$ or $\mathbb{C}$, of dimension $n-r$, and let $f: X \rightarrow$ $k^{m}$ be a polynomial dominant mapping. In the seventies Wallace [19], Varchenko [17] and Verdier [18] proved that there exists a proper algebraic (or semialgebraic in the real case) set $B \subset k^{m}$ such that

$$
f: X \backslash f^{-1}(B) \rightarrow k^{m} \backslash B
$$

is a locally trivial $C^{\infty}$ fibration. We call the smallest such $B$ the bifurcation set of $f$ and we denote it by $B(f)$. In a natural way a question of how to describe this set appears.

Since, in general, $f$ is non-proper the set $B(f)$ is larger than $K_{0}(f)$ - the set of critical values of $f$. It also contains the set $B_{\infty}(f)$ of bifurcation points at infinity. Briefly speaking the set $B_{\infty}(f)$ consists of points at which $f$ is not a locally trivial fibration at infinity (i.e., outside a compact set). The main difficulty is to understand the set $B_{\infty}(f)$. The usual way is to apply the stratification theory to the projective closure of the graph of $f$. However, it is very difficult by this method to give an explicit equation for $B_{\infty}(f)$ or even for a hypersurface which contains $B_{\infty}(f)$.

We follow another approach which in fact goes back to Ehresemann, Palais and Malgrange and was developed by Rabier [16]. To control the set $B_{\infty}(f)$ we use the set of asymptotic critical values at infinity of $f[16]$ :

$$
K_{\infty}(f)=\left\{y \in k^{m}: \exists_{x_{l} \in X,\left|x_{l}\right| \rightarrow \infty} \text { s.t. } f\left(x_{l}\right) \rightarrow y \text { and }\left|x_{l}\right| v\left(d_{x_{l}} f\right) \rightarrow 0\right\},
$$

where $v$ stands for the distance of $d_{x_{l}} f$ to the space of degenerate linear maps on the tangent space to $X$ at $x_{l}$. In the next section we explain different ways to compute $v$.

We say that $K(f)=K_{0}(f) \cup K_{\infty}(f)$ is the set of generalized critical values of $f$. It follows from a general result of Rabier [16] that $B_{\infty}(f) \subset K_{\infty}(f)$. Hence $B(f) \subset K(f)$ which means that $f$ is a locally trivial fibration over the complement of $K(f)$. The question when $B(f)=K(f)$ was studied (among others) by Parusiński [14].

In Section 3 we prove that $K(f)$ is a semialgebraic subset of $k^{m}$ of measure zero. This generalizes the result from [12], where the case of polynomial mappings from $\mathbb{C}^{n}$ to $\mathbb{C}^{m}$ was studied.

In Section 4 we prove that in the complex case $K(f)$ is actually a proper algebraic subset of $\mathbb{C}^{m}$. In fact we give an explicit description of $K(f)$ which allows us to estimate from above the degree of a hypersurface containing $K(f)$.

More precisely let $X \subset \mathbb{C}^{n}$ be a smooth affine variety of dimension $n-r$. Let $f=\left(f_{1}, \ldots, f_{m}\right): X \rightarrow \mathbb{C}^{m}$ be a polynomial dominant mapping. Assume that $I(X)$ the ideal of $X$-is generated by polynomials of degree at most $D$ and $\operatorname{deg} f_{i} \leq d$.

Our main result, Theorem 4.1, claims that $K(f)$ is contained in an algebraic hypersurface of degree at most

$$
\begin{aligned}
(d+(m-1) & (d-1)+(D-1) r)^{n-r}(\operatorname{deg} X) \\
& \leq(d+(m-1)(d-1)+(D-1) r)^{n-r} D^{r} .
\end{aligned}
$$

This generalizes (and slightly improves) the result from [8], where the case of polynomial mappings from $\mathbb{C}^{n}$ to $\mathbb{C}^{m}$ was studied. Note that technically it is much harder to describe 
$K_{\infty}(f)$ when we have to use equations for $X$. It follows from Theorem 4.1 that if $X$ is a hypersurface of degree $D$ and $f: X \rightarrow \mathbb{C}$ is a polynomial of degree $d$, then $f$ has at most $(d+D-1)^{n-1} D$ generalized critical values. Hence in this case we have that all fibers of $f$ are smooth and diffeomorphic, with at most $(d+D-1)^{n-1} D$ exceptions.

In a particularly interesting case of linear mapping (projections on line) this gives a bound $D^{n}$ for the number of generalized critical values, hence for the number of atypical values. We give an example which shows this bound is asymptotically optimal. Moreover, we obtain similar results in the real case.

In Section 5 we give an algorithm to compute the set $K(f)$ effectively. All necessary results from linear algebra are given in Section 2 . In particular we explain geometrically and analytically how to compute $v$-the distance to singular operators-moreover, we give several equivalent expressions for $v$.

\section{Preliminaries}

Let $k=\mathbb{R}$ or $k=\mathbb{C}$. Let $X \cong k^{n}, Y \cong k^{m}$ be finite-dimensional vector spaces (over $k)$. We consider those spaces equipped with the canonical scalar (hermitian) products. We denote by $\mathcal{L}(X, Y)$ the set of linear mappings from $X$ to $Y$ and by $\Sigma=\Sigma(X, Y) \subset$ $\mathcal{L}(X, Y)$ the set of non-surjective mappings. In this section we give several different expressions for the distance of an $A \in \mathcal{L}(X, Y)$ to the space $\Sigma$ of singular operators. Let us first recall the following [16]:

Definition 2.1. Let $A \in \mathcal{L}(X, Y)$. Set

$$
v(A)=\inf _{\|\varphi\|=1}\left\|A^{*}(\varphi)\right\|,
$$

where $A^{*}: \mathcal{L}\left(Y^{*}, X^{*}\right)$ is adjoint operator and $\varphi \in Y^{*}$.

Remark 2.1. Recall (see [16]) that if $A \in G L(X, Y)$, then $v(A)=\left\|A^{-1}\right\|^{-1}$.

Moreover in [12] we have a following useful characterizations of $v(A)$ :

Proposition 2.1. Let $A \in \mathcal{L}(X, Y)$. Denote $B_{X}(0,1)=\{x \in X ;|x| \leq 1\}$ and $B_{Y}(0, r)=\{y \in Y ;|y| \leq r\}$. Then

(a) $v(A)=\sup \left\{r>0: B_{Y}(0, r) \subset A\left(B_{X}(0,1)\right)\right\}$.

(b) $v(A)=\operatorname{dist}(A, \Sigma)=\inf _{B \in \Sigma}\|A-B\|$.

Let $\alpha, \beta: \mathcal{L}(X, Y) \rightarrow \mathbb{R}_{+}$be two non-negative functions. We say that $\alpha$ and $\beta$ are equivalent (we write $\alpha \sim \beta$ ) if there are constants $c, d>0$ such that

$$
c \alpha(A) \leq \beta(A) \leq d \alpha(A)
$$

for any $A \in \mathcal{L}(X, Y)$. We give below several functions equivalent to $v$. Let $A=$ $\left(A_{1}, \ldots, A_{m}\right) \in \mathcal{L}(X, Y)$ and let $\overline{A_{i}}=\operatorname{grad} A_{i}$. Denote by $\left\langle\left(\overline{A_{j}}\right)_{j \neq i}\right\rangle$ the linear space generated by vectors $\left(\overline{A_{j}}\right), j \neq i$. Let

$$
\kappa(A)=\min _{1 \leq i \leq m} \operatorname{dist}\left(\overline{A_{i}},\left\langle\left(\overline{A_{j}}\right)_{j \neq i}\right\rangle\right)
$$

be the Kuo number of $A$. 
Proposition 2.2 [12]. The Kuo function $\kappa$ is equivalent to $v$ of Rabier. More precisely

$$
v(A) \leq \kappa(A) \leq \sqrt{m} v(A)
$$

Definition 2.2. Let $A \in \mathcal{L}(X, Y)$ and let $H \subset X$ be a linear subspace. We set

$$
v(A, H)=v\left(\left.A\right|_{H}\right), \quad \kappa(A, H)=\kappa\left(\left.A\right|_{H}\right),
$$

where $\left.A\right|_{H}$ denotes the restriction of $A$ to $H$.

From Proposition 2.2 we immediately get:

Corollary 2.1. We have $v(A, H) \sim \kappa(A, H)$.

In fact we also have an explicit expression for $\kappa(A, H)$ :

Proposition 2.3. Let $A=\left(A_{1}, \ldots, A_{m}\right) \in \mathcal{L}(X, Y)$ and let $H \subset X$ be a linear subspace. Assume that $H$ is given by a system of linear equations $B_{j}=0, j=1, \ldots, r$. Then

$$
\kappa(A, H)=\min _{1 \leq i \leq m} \operatorname{dist}\left(\overline{A_{i}},\left\langle\left(\overline{A_{j}}\right)_{j \neq i} ;\left(\overline{B_{j}}\right)_{j=1, \ldots, r}\right\rangle\right),
$$

where $\overline{A_{i}}=\operatorname{grad} A_{i}$ and $\overline{B_{j}}=\operatorname{grad} B_{j}$.

Proof. The space $B=\left\langle\left(\overline{B_{j}}\right)_{j=1, \ldots, r}\right\rangle$ is the orthogonal supplement to $H$. Hence every vector $\overline{A_{i}}$ can be written as $a_{i}+b_{i}$, where $a_{i} \in H, b_{i} \in B$. Thus $\operatorname{dist}\left(\overline{A_{i}},\left\langle\left(\overline{A_{j}}\right)_{j \neq i} ; B\right\rangle\right)=$ $\operatorname{dist}\left(a_{i},\left\langle\left(a_{j}\right)_{j \neq i}\right\rangle\right)$ and since $\operatorname{grad}\left(A_{i} \mid H\right)=a_{i}$ the proof is finished.

Finally we introduce function $g^{\prime}$, which will be useful in the explicit description of the set of generalized critical values:

Definition 2.3. Let $A \in \mathcal{L}\left(k^{n}, k^{m}\right)$, where $n \geq m+r$, and let $H \subset k^{n}$ be a linear subspace given by a system of independent linear equations $B_{l}=\sum b_{l k} x_{k}, l=1, \ldots, r$. By abuse of notation we denote by $A$ the matrix (in the canonical bases in $k^{n}$ and $k^{m}$ ) of the mapping $A$. Let $C=$ be an $(m+r) \times n$ matrix given by rows $A_{1}, \ldots, A_{m} ; B_{1}, \ldots, B_{r}$ (we identify $A_{i}=\sum a_{i k} x_{k}$ with the vector $\left(a_{j 1}, \ldots, a_{j n}\right)$, similarly for $\left.B_{l}\right)$. Let $M_{I}$, where $I=\left(i_{1}, \ldots, i_{m+r}\right)$, denote an $((m+r) \times(m+r))$ minor of $C$ given by columns indexed by $I$. Let $M_{J}(j)$ denote an $(m+r-1) \times(m+r-1)$ minor given by columns indexed by $J$ and by deleting the $j$ th row, where $1 \leq j \leq m$. Note that we delete only $A_{j}$ rows! We set

$$
g^{\prime}(A, H)=\max _{I}\left\{\min _{\{J \subset I, 1 \leq j \leq m\}} \frac{\left|M_{I}\right|}{\left|M_{J}(j)\right|}\right\},
$$

(where we consider only numbers with $M_{J}(j) \neq 0$, if all numbers $M_{J}(j)$ are zero, we put $\left.g^{\prime}(A)=0\right)$.

In particular we get the following: 
Proposition 2.4. We have $g^{\prime}(A, H) \sim v(A, H)$.

First we prove

Lemma 2.1. Let $H$ be a linear subspace of $k^{n}, \operatorname{dim} H=p$, then there exists a coordinate linear subspace $E, \operatorname{dim} E=p$, such that

$$
v\left(\left.\pi\right|_{H}\right) \geq\left(\begin{array}{l}
n \\
p
\end{array}\right)^{-1 / 2}
$$

where $\left.\pi\right|_{H}$ is the orthogonal projection on $E$ restricted to $H$.

Proof. Recall that the canonical scalar (Hermitian) product on $k^{n}$ induces a scalar (Hermitian) product on $\wedge^{p} k^{n}$, see, e.g., [3]. If $x=x_{1} \wedge \cdots \wedge x_{p}$ and $y=y_{1} \wedge \cdots \wedge y_{p}$, then we put

$$
(x \mid y)=\operatorname{det}\left(x_{i} \mid y_{j}\right)_{i, j=1, \ldots, n} .
$$

Let $e_{1}, \ldots, e_{n}$ be the canonical basis of $k^{n}$ and let $I=\left(i_{1}, \ldots, i_{p}\right)$ be an multiindex such that $1 \leq i_{1}<\cdots<i_{p} \leq n$. Denote $e_{I}=e_{i_{1}} \wedge \cdots \wedge e_{i_{p}}$, then all $e_{I}$ form an orthonormal basis of $\wedge^{p} k^{n}$. Let us choose some orthonormal basis $f_{1}, \ldots,, f_{p}$ of $H$ and put $f=f_{1} \wedge \cdots \wedge f_{p}$. Clearly, $f=\sum a_{I} e_{I}$, but $\|f\|=1$, so

$$
1=\sum_{I}\left|a_{I}\right|^{2}
$$

We have $\left(\begin{array}{l}n \\ p\end{array}\right)$ positive summands, hence there is at least one $I_{0}$ such that $\left|a_{I_{0}}\right| \geq\left(\begin{array}{l}n \\ p\end{array}\right)^{-1 / 2}$.

We take as $E$ the vector space generated by $e_{i}, i \in I_{0}$. Note that the Jacobian of the orthogonal projection $\left(\left.\pi\right|_{H}\right)$ of $H$ on $E$ is exactly $a_{I_{0}}$. Let $B$ be the unit ball in $H$, its image is an ellipsoid with semiaxes $0<b_{1} \leq b_{2} \leq \cdots \leq b_{p} \leq 1$. By the classical change of variables formula we see that the volume of $\pi(B)$ equals $\left|a_{I_{0}}\right|$ times volume of $B$. Hence

$$
b_{1} b_{2} \cdots b_{p}=\left|a_{I_{0}}\right|,
$$

and consequently $b_{1} \geq\left|a_{I_{0}}\right|$. It is an immediate consequence of Proposition 2.1 that $b_{1}=v\left(\left.\pi\right|_{H}\right)$. Hence the lemma follows.

Suppose that $v(A, H)>0$. We fix $I=\left(i_{1}, \ldots, i_{m+r}\right)$ and assume that $M_{J}(j) \neq 0$ for all $\{J \subset I, 1 \leq j \leq m\}$. Note that by Lemma 2.1 such an $I$ exists. Let $E$ be the subspace generated by $e_{i}, i \in I$, and let $\pi$ be the orthogonal projection on $E$. By Lemma 2.1 we may assume that $v\left(\left.\pi\right|_{H}\right) \geq \delta$, where $\delta=\left(\begin{array}{c}n \\ m+r\end{array}\right)^{-1 / 2}$. Let $g=v\left(\left.\pi\right|_{H}\right)^{-1}$, note that

$$
\|g\| v\left(\left.A\right|_{H}\right) \geq v\left(\left(\left.A\right|_{H}\right) \circ g\right) \geq v\left(\left.A\right|_{H}\right) v(g)
$$

and recall that $v\left(\left.\pi\right|_{H}\right)=\|g\|^{-1}$. So it is enough to study $\left(\left.A\right|_{H}\right) \circ g$, i.e., the matrix obtained from $A$ by deleting columns which are not in $I$. Thus we may assume that $n=m+r$. By Remark 2.1, $v(A, H)=\|h\|^{-1}$, where $h=\left(\left.A\right|_{H \cap \mathrm{ker}^{\perp}}\right)^{-1}$. Again by Lemma 2.1 we may consider the composition of $h$ with the orthogonal projection 
on some coordinate $m$-plane, say generated by the first $m$ coordinates. Recall that the mapping $C: k^{n} \rightarrow k^{m+r}$ is invertible. By the formula for the inverse matrix (or directly by Cramer's rule) we can see that there are constants $\alpha, \beta>0$ such that

$$
\alpha\|h\|^{-1} \leq\left(\max _{\{J \subset I, 1 \leq j \leq m\}} \frac{\left|M_{J}(i)\right|}{\left|M_{I}\right|}\right) \leq \beta\|h\|^{-1},
$$

which proves Proposition 2.4.

We end this section by giving another equivalent expression for $v$ (which will be useful in the proof of Theorem 4.1).

Definition 2.4. With the notation of Definition 2.3 we put

$$
q(A, H)=\frac{\max _{I}\left|M_{I}\right|}{\max _{I, J \subset I, j}\left|M_{J}(j)\right|},
$$

where we consider only numbers with $M_{J}(j) \neq 0$, if all numbers $M_{J}(j)$ are zero, we put $q(A)=0$.

We leave the proof of the following fact to the reader.

Proposition 2.5. The function $q(A, H)$ is equivalent to $v(A, H)$.

\section{Rabier's Fibration Theorem and the Sard Theorem for $K(f)$}

Definition 3.1. Let $k=\mathbb{C}$ or $k=\mathbb{R}$ and let $X$ be a smooth affine variety over $k$. Let $f: X \rightarrow k^{m}$ be a $k$-smooth mapping. Recall that we define the set of generalized critical values as

$$
K_{\infty}(f)=\left\{y \in k^{m}: \exists_{x_{l} \in X,\left|x_{l}\right| \rightarrow \infty} \text { s.t. } f\left(x_{l}\right) \rightarrow y \text { and }\left|x_{l}\right| \nu d\left(f\left(x_{l}\right)\right) \rightarrow 0\right\} .
$$

Remark 3.1. Note that by virtue of the results of Section 2 we can replace the function $\nu$ by an arbitrary function among $\kappa, g, g^{\prime}$.

We also have an easy but important observation:

Remark 3.2. Recall that we define by $K_{0}(f)$ the set of critical values of $f$. Let $k=\mathbb{C}$ or $k=\mathbb{R}$ and let $X$ be a smooth affine variety over $k$. Let $f: X \rightarrow k^{m}$ be a $k$-smooth mapping. Then the set $K(f)=K_{0}(f) \cup K_{\infty}(f)$ is closed.

Now we state the basic theorem which follows from the main result of Rabier [16]:

Theorem 3.1. Let $k=\mathbb{C}$ or $k=\mathbb{R}$ and let $X$ be a smooth affine variety over $k$ of dimension $n-r \geq m$. Let $f: X \rightarrow k^{m}$ be a $k$-smooth mapping. Then

$$
f: X \backslash f^{-1}(K(f)) \rightarrow k^{m} \backslash K(f)
$$

is a locally trivial fibration. 
Recall that a value $y$ of the map $f$ is called typical if $f$ is a $C^{\infty}$ fibration (possibly with empty fiber) over a neighborhood of $y$ and atypical otherwise. Note that a typical value is not necessarily a value of $f$ ! The set $B(f)$ is called the bifurcation set of $f$. Rabier's theorem [16] states that

$$
B(f) \subset K(f)=K_{0}(f) \cup K_{\infty}(f) .
$$

A short, direct proof of the finite-dimensional case of the Rabier theorem (which is what we actually need) is contained also in a note by Jelonek [9].

It is crucial to know that the set $K(f)$ is small, in particular that this set is nowhere dense. In [12] it was proven that for a polynomial mapping $f: k^{n} \rightarrow k^{m}$, the set $K(f)$ is semialgebraic of dimension less than $m$ in the case $k=\mathbb{R}$, and algebraic of complex dimension less than $m$ in the case $k=\mathbb{C}$. We give below a proof of this fact in the case of mapping $f: X \rightarrow k^{m}$, where $X$ is smooth algebraic. We follow the idea from [12] simplifying it at some points.

Before we state the main result of this section we need some additional results. We need the fact, due to Kurdyka [11], that any semialgebraic set $A \subset \mathbb{R}^{n}$ is a finite union $A=\bigcup_{i} L^{i}$, where each $L^{i}$ has the Whitney property with constant $M$ : any two points $x, y \in L^{i}$ can be joined in $L^{i}$ by a piecewise smooth arc of length $\leq M|x-y|$. What we actually need is a uniform version of the above decomposition, for families parameterized by finite-dimensional spaces: if $B \subset \mathbb{R}^{n} \times \mathbb{R}^{p}$ and $t \in \mathbb{R}^{p}$, we write $B_{t}=\left\{x \in \mathbb{R}^{n}:(x, t) \in B\right\}$. Then from the method of [11], we obtain the following theorem:

Theorem 3.2. There exists $M=M(n)>0$ such that any semialgebraic set $A \subset$ $\mathbb{R}^{n} \times \mathbb{R}^{p}$ can be decomposed into a finite (and disjoint) union $A=\bigcup_{i \in I} L^{i}$, such that for each $t \in \mathbb{R}^{p}$, every set $L_{t}^{i}$ has the Whitney property with constant $M$. So, in particular $A_{t}=\bigcup_{i \in I} L_{t}^{i}$ for each $t \in \mathbb{R}^{p}$. (Clearly, for a fixed $t \in \mathbb{R}^{p}$ some of $L_{t}^{i}$ may be empty.)

Further we shall use the following version of the curve selection lemma for semialgebraic sets (it can be easily obtained using a semialgebraic compactification of $\mathbb{R}^{n}$ and the classical curve selection lemma, see [2] and [1]).

Lemma 3.1 (Curve Selection at Infinity). Let $A \subset \mathbb{R}^{n}$ and let $\varphi: A \rightarrow \mathbb{R}^{q}$ be a semialgebraic map. Assume that there exists a sequence $x_{l} \in A$ such that $\left|x_{l}\right| \rightarrow \infty$ and $\varphi\left(x_{l}\right) \rightarrow y$, for some $y \in \mathbb{R}^{q}$. Then there exists a semialgebraic arc $\gamma:[\alpha, \beta) \rightarrow \mathbb{R}^{n}$ such that $\gamma(t) \in A, \lim _{t \rightarrow \beta}|\gamma(t)|=+\infty$ and $\lim _{t \rightarrow \beta} \varphi(\gamma(t))=y$.

Now consider such a semialgebraic arc $\gamma:(\alpha, \beta) \rightarrow \mathbb{R}^{n}$. Since $\left|\gamma^{\prime}(t)\right|>0$ for $t$ close to $\beta$, we may reparametrize $\gamma$ in such a way that $\beta=+\infty$ and $|\gamma(r)|=r$. Under this assumption we have:

Lemma 3.2. $\lim _{r \rightarrow \infty}\left|\gamma^{\prime}(r)\right|=1$; in particular, $\gamma^{\prime}(r)$ is bounded for $r>0$ large enough.

Proof. Since $\gamma$ is semialgebraic, $\lim _{r \rightarrow \infty}(\gamma(r) /|\gamma(r)|)$ and $\lim _{r \rightarrow \infty}\left(\gamma^{\prime}(r) /\left|\gamma^{\prime}(r)\right|\right)$ exist. Hence, it is easily seen that these limits are equal. In other words, $\cos \theta(r) \rightarrow 1$, as 
$r \rightarrow \infty$, where $\theta(r)$ is the angle between $\gamma(r) /|\gamma(r)|=\gamma(r) /|r|$ and $\gamma^{\prime}(r) /\left|\gamma^{\prime}(r)\right|$. Differentiation of $|\gamma(r)|^{2}=r^{2}$ yields $\left|\gamma^{\prime}(r)\right|=1 / \cos \theta(r)$. This implies the lemma.

In order to prove our Sard theorem, we also use the fact that for a fixed mapping $f$, the convergence of $v\left(d f\left(x_{l}\right)\right)$ in the definition of $K_{\infty}(f)$ is actually faster than $\left|x_{l}\right|^{-1}$. To make this precise, for a differentiable function $f: \mathbb{R}^{n} \rightarrow \mathbb{R}^{m}$ and any $N \in \mathbb{N}^{*}$ we define

$K_{\infty}^{N}(f)=\left\{y \in \mathbb{R}^{k}: \exists x_{l} \in \mathbb{R}^{n},\left|x_{l}\right| \rightarrow \infty\right.$ s.t. $f\left(x_{l}\right) \rightarrow y$ and $\left.\left|x_{l}\right|^{1+1 / N} v\left(d f\left(x_{l}\right)\right) \rightarrow 0\right\}$.

We have

Lemma 3.3. Let $f: X \rightarrow \mathbb{R}^{m}$ be a differentiable semialgebraic function. Then

$$
K_{\infty}(f)=\bigcup_{N=1}^{\infty} K_{\infty}^{N}(f) .
$$

Proof. Let $y \in K_{\infty}(f)$. By Lemma 3.1 there is a semialgebraic arc $\gamma:[\alpha, \beta) \rightarrow X$, such that $\lim _{t \rightarrow \beta}|\gamma(t)|=+\infty, \lim _{t \rightarrow \beta}|\gamma(t)| v(d f(\gamma(t)))=0$ and $\lim _{t \rightarrow \beta} \varphi(\gamma(t))=y$. We consider semialgebraic functions $A(t)=|\gamma(t)| v(d f(\gamma(t)))$ and $B(t)=1 /|\gamma(t)|$ defined on $[\alpha, \beta]$. We can assume that $z \operatorname{eros}(B)=z \operatorname{eros}(A)=\{\beta\}$, hence by the Lojasiewicz inequality (see Theorem 2.3 .11 on p. 63 of [1]), there is a constant $c>$ 0 and the integer $n>0$ such that $A \leq c B^{1 / n}$. Consequently, for $N>n$ we have $\lim _{t \rightarrow \beta}|\gamma(t)|^{1+1 / N} v(d f(\gamma(t)))=0$. Thus $y \in K_{\infty}^{N}(f)$.

The aim of this section is to prove the following:

Theorem 3.3. Let $X \subset k^{n}$ be an affine variety of dimension $n-r$. Let $f: X \rightarrow k^{m}$ be a polynomial map. Then $K(f)$ is a closed semialgebraic set of a Lebesgue measure 0 . In particular it is of dimension less than $m$.

Proof. We can assume that $k=\mathbb{R}$. Clearly $K_{\infty}(f)$ is a semialgebraic subset of $\mathbb{R}^{m}$. By Lemma 3.3 and the basic properties of the Lebesgue measure it is enough to prove that $K_{\infty}^{N}(f)$ is of measure zero for any integer $N>0$.

Let $I(X)$ denote the ideal of functions vanishing on $X$, assume that $I(X)$ is generated by polynomials $b_{1}, \ldots, b_{w}$. Since the variety $X$ can be covered by at most $p=\left(\begin{array}{l}w \\ r\end{array}\right)$ Zariski-open subsets in which it is a complete intersection, we can assume that $X$ is a complete intersection, that is $w=r$. So in particular if $x \in X$, then $T_{x} X=\bigcap_{j=1}^{r}\left\{d_{x} b_{j}=\right.$ $0\}$. We write $f=\left(f_{1}, \ldots, f_{m}\right)$ for components of $f$, where $f_{i}: \mathbb{R}^{n} \rightarrow \mathbb{R}$ is a polynomial.

By Proposition 2.2, we may replace the distance $v$ of Rabier by the distance $\kappa\left(d_{x} f, T_{x} X\right)$ of Kuo. For each $i \in\{1, \ldots, m\}$, we define

$$
D_{i}=\left\{x \in X: \kappa\left(d f(x), T_{X}\right)=\operatorname{dist}\left(\nabla f_{i}(x), V_{i}(x)\right)\right\},
$$


where $V_{i}(x)$ is the vector space generated by $\nabla f_{j}(x), j=1, \ldots, m, j \neq i$, and by $\nabla b_{j}(x), j=1, \ldots, r$. Clearly each $D_{i}$ is semialgebraic in $\mathbb{R}^{n}$ and $X=\bigcup_{i=1}^{m} D_{i}$, so

$$
K_{\infty}(f)=\bigcup_{i=1}^{m} K_{\infty}\left(f_{\mid D_{i}}\right)
$$

where

$K_{\infty}\left(f_{\mid D_{i}}\right)=\left\{y \in \mathbb{R}^{m}: \exists x_{l} \in D_{i},\left|x_{l}\right|^{1+1 / N} \rightarrow \infty, f\left(x_{l}\right) \rightarrow y\right.$ and $\left.\left|x_{l}\right| v\left(d f\left(x_{l}\right)\right) \rightarrow 0\right\}$.

We shall prove the following:

Lemma 3.4. For each $i \in\{1, \ldots, k\}$ we have vol $_{m}\left(K_{\infty}\left(f_{\mid D_{i}}\right)\right)=0$. In particular $\operatorname{dim} K_{\infty}(f)<m$.

Proof. We give the proof for $i=1$, we write $D=D_{1}, \bar{f}=\left(f_{2}, \ldots, f_{m}\right)$.

We fix $B$ is an open ball in $\mathbb{R}^{m-1}$ and $(\alpha, \beta)$ is an open bounded interval in $\mathbb{R}$. The lemma is clearly a consequence of

$$
\operatorname{vol}_{m}\left(K_{\infty}\left(f_{\mid D}\right) \cap(\alpha, \beta) \times B\right)=0 .
$$

In order to prove equality (3.1), we construct a family of sets $\Delta_{r}$ such that

$$
\overline{\Delta_{r}} \supset K_{\infty}\left(f_{\mid D}\right) \cap(\alpha, \beta) \times B \quad \text { and } \quad \operatorname{vol}_{m}\left(\overline{\Delta_{r}}\right) \rightarrow 0 \quad \text { as } \quad r \rightarrow \infty .
$$

We first define

$$
\widetilde{\Sigma}_{r}=\left\{x \in D:|x| \geq r, f_{1}(x) \in(\alpha, \beta), \bar{f}(x) \in B \text { and }|x|^{1+1 / N} \kappa(d f(x)) \leq 1\right\},
$$

where $r>0$, and put

$$
\Delta_{r}=f\left(\widetilde{\Sigma}_{r}\right)
$$

and finally

$$
\Delta=\bigcap_{r>0} \bar{\Delta}_{r} .
$$

Every $\Delta_{r}$ is semialgebraic, hence we have $\operatorname{vol}_{m}\left(\bar{\Delta}_{r}\right)=\operatorname{vol}_{m}\left(\Delta_{r}\right)$ and consequently

$$
\operatorname{vol}_{m}(\Delta)=\lim _{r \rightarrow \infty} \operatorname{vol}_{m}\left(\Delta_{r}\right)
$$

since the family $\left(\Delta_{r}\right)_{r>0}$ is decreasing with respect to $r \rightarrow \infty$.

It is clear that

$$
K_{\infty}\left(f_{\mid D}\right) \cap(\alpha, \beta) \times B \subset \Delta,
$$

so it is enough to prove that $\operatorname{vol}_{m}(\Delta)=0$. First, using Fubini's theorem, we write

$$
\operatorname{vol}_{m}\left(\Delta_{r}\right)=\int_{B} \mu_{r}(b) d b,
$$


where $d b$ stands for the Lebesgue measure on $\mathbb{R}^{m-1}$, and

$$
\mu_{r}(b)=\operatorname{vol}_{1}\left(\left\{y_{1} \in \mathbb{R}:\left(y_{1}, b\right) \in \Delta_{r}\right\}\right)
$$

Clearly, each $\mu_{r}$ is measurable. Moreover, for fixed $b \in B$, the function $r \mapsto \mu_{r}(b) \geq$ 0 is decreasing. Let

$$
\mu(b)=\lim _{r \rightarrow \infty} \mu_{r}(b) .
$$

By Lebesgue's theorem on bounded convergence, we obtain

$$
\operatorname{vol}_{m}(\Delta)=\int_{B} \mu(b) d b .
$$

Now the final point in the proof of equality (3.1) is the fact that $m \equiv 0$, which follows from the next lemma.

Lemma 3.5. There exists a constant $c>0$ such that, for $r$ large enough,

$$
\mu_{r}(b) \leq c r^{-1 / N}
$$

Proof. To prove Lemma 3.5, we introduce the semialgebraic family

$$
\Sigma_{r, b}=\widetilde{\Sigma}_{r} \cap \bar{f}^{-1}(b) \cap S(r),
$$

where $b \in B, r>0$, and next we write

$$
\widetilde{\Sigma}_{r, b}=\widetilde{\Sigma}_{r} \cap \bar{f}^{-1}(b)=\bigcup_{s \geq r} \Sigma_{s, b} .
$$

Note that

$$
\mu_{r}(b)=\operatorname{vol}_{1}\left(f_{1}\left(\widetilde{\Sigma}_{r, b}\right)\right) .
$$

It follows from Theorem 3.2 that there exists a finite family $L^{i} \subset X \times \mathbb{R} \times \mathbb{R}^{m-1}, i \in I$, of semialgebraic sets such that

$$
\Sigma_{r, b}=\bigcup_{i \in I} L_{r, b}^{i}
$$

Each $L_{r, b}^{i}$ has the Whitney property with constant $M$ (some of $L_{r, b}^{i}$ may be empty).

Recall that the condition $|x|^{1+1 / N} \kappa(d f(x)) \leq 1$ for $x \in \bar{f}^{-1}(b)=W_{b}$ means that

$$
\left|\nabla f_{1 \mid W_{b}}(x)\right| \leq|x|^{-(1+1 / N)} \text {. }
$$

Hence, by the mean value theorem $f_{1}\left(L_{r, b}^{i}\right)$ is a segment of length $d(r)$ where

$$
d(r) \leq 2 M r \sup _{L_{r, b}^{i}}\left|\nabla f_{1 \mid W_{b}}\right| \leq 2 M r^{-1 / N}
$$

Fix $b \in B, i \in I$ and assume that $L_{r, b}^{i} \neq \emptyset$ for any $r$ large enough. Applying the curve selection lemma at infinity, we obtain a semialgebraic arc $\gamma:[r,+\infty) \rightarrow \mathbb{R}^{n}$ such that $\gamma(\zeta) \in L_{\zeta, b}^{i}$. In particular, $\gamma(\zeta) \in \bar{f}^{-1}(b)=W_{b}$ and $|\gamma(\zeta)|=\zeta$. 
By Lemma 3.2, we may suppose that $\left|\gamma^{\prime}(\zeta)\right| \leq 2$. So we can easily compute the length of $f_{1} \circ \gamma([r,+\infty))$; namely, by (3.2) we have

$$
\int_{r}^{+\infty}\left|\left(f_{1} \circ \gamma\right)^{\prime}(\zeta)\right| d \zeta \leq 2 \int_{r}^{+\infty} \zeta^{-(1+1 / N)} d \zeta=2 N r^{-1 / N} .
$$

Thus, by (3.3) and (3.4), $f_{1}\left(\bigcup_{\zeta \geq r} L_{\zeta, b}^{i}\right)$ is contained in a segment of length

$$
(4 M+2 N) r^{-1 / N} .
$$

Therefore $f_{1}\left(\widetilde{\Sigma}_{r, b}\right)$ is contained in \#I segments of this length. Put $c=(\# I)(4 M+2 N)$; we have

$$
\mu_{r}(b) \leq c r^{-1 / N}
$$

and Lemma 3.5 follows.

Finally from Lemma 3.4 and the usual semialgebraic Sard's theorem (see [1]) it follows that $\operatorname{dim} K(f)<m$.

\section{Estimates for the Degree}

In the proof of our next theorem we need the following technical lemmas.

Lemma 4.1. Let $A$ be algebraic subsets of $\mathbb{C}^{N}, \operatorname{dim} A=n$. Let $L, M$ be linear subspaces of $\mathbb{C}^{N}$, and $L \subset M$. Let $\operatorname{dim} M=n+1$. Assume that $L \not \subset A$. Then there exists a linear projection $p: \mathbb{C}^{N} \rightarrow M$ such that $p$ restricted to $A$ is finite and $L \not \subset p(A)$.

Proof. Take a point $a \in L \backslash A$. Let $\Lambda$ be the Zariski closure of the cone $\bigcup \overline{a x}, x \in A$. It is easy to see that $\operatorname{dim} \Lambda \leq n+1$. Let $H_{\infty}$ be the hyperplane at infinity of $\mathbb{C} \times \mathbb{C}^{N}$. For any $Z \subset \mathbb{C} \times \mathbb{C}^{N}$ denote by $\tilde{Z}$ the projective closure of $Z$. Observe that

$$
\operatorname{dim} H_{\infty} \cap(\tilde{\Lambda} \cup \tilde{\Gamma} \cap \tilde{M}) \leq n .
$$

Thus, there is a projective subspace $Q \subset H_{\infty}$ of dimension $N-n-2$, which is disjoint with $(\tilde{\Lambda} \cup \tilde{A} \cap \tilde{M})$. Denote by $p_{Q}: \mathbb{P}^{N} \backslash Q \rightarrow \tilde{M}$ the linear projection determined by the subspace $Q$.

Now, let $p: A \rightarrow M$ be the restriction of $p_{Q}$ to $A$. It is easily seen that $p$ has desired properties, i.e., $p: A \rightarrow M$ is a finite mapping and $a \notin L \cap p(A)$.

Lemma 4.2. Let $F=\left(f_{1}, \ldots, f_{n}, f_{n+1}\right): X-\rightarrow \mathbb{C}^{n+1}$ be a rational mapping. Assume that $\Gamma:=\operatorname{cl}\left(F\left(\mathbb{C}^{n}\right)\right)$ is a hypersurface. Let $f_{i}=P_{i} / G$, where $\operatorname{deg} P_{i}=d_{i}$ and $\operatorname{deg} G=d_{0}$. Take $d=\max _{0 \leq i \leq n+1}\left\{d_{i}\right\}$. Then

$$
\operatorname{deg} \Gamma \leq d^{n} \operatorname{deg} X / \mu(F),
$$

where $\mu(F)=\left(\mathbb{C}(X)\right.$ : $\left.\mathbb{C}\left(f_{1}, \ldots, f_{n+1}\right)\right)$. 
Proof. We can estimate the degree of $\Gamma$ using the Bezout theorem. Indeed, the number $(\operatorname{deg} \Gamma) \mu(F)$ is estimated by the number of solution of a generic system of equations:

$$
\sum_{j=1}^{n+1} a_{i j} f_{j}(x)=c_{i}, \quad i=1, \ldots, n, \quad x \in X .
$$

This system is equivalent to the system

$$
\sum_{j=1}^{n+1} a_{i j} P_{j}(x)=c_{i} G(x), \quad i=1, \ldots, n, \quad x \in X,
$$

and by the Bezout theorem we have $\mu(F) \operatorname{deg} \Gamma \leq d^{n} \operatorname{deg} X$.

Theorem 4.1. Let $X \subset \mathbb{C}^{n}$ be a smooth affine variety of dimension $n-r \geq m$. Assume that $I(X)=\left\{b_{1}, \ldots, b_{w}\right\}$, where deg $b_{i} \leq D$. Let $f=\left(f_{1}, \ldots, f_{m}\right): X \rightarrow \mathbb{C}^{m}$ be a polynomial dominant mapping and let $\operatorname{deg} f_{i} \leq d$. Then the set $K(f)$ is a proper algebraic subset of $\mathbb{C}^{m}$ and is contained in a hypersurface of degree at most

$(d+(m-1)(d-1)+(D-1) r)^{n-r} \operatorname{deg} X \leq(d+(m-1)(d-1)+(D-1) r)^{n-r} D^{r}$.

Proof. Since $X$ is smooth it means that there exists an open dense subset $U \subset X$, on which $X$ is a complete intersection. As follows from the rest of the proof, we can assume, without loss of generality, that $X=U$, i.e., that $X$ is a complete intersection.

Recall the notation of Definition 2.3. For $x \in \mathbb{C}$ let $A=d_{x} f$, and $B_{l}=d_{x} b_{l}$, $l=1, \ldots, r$. Let $A \in \mathcal{L}\left(k^{n}, k^{m}\right)$, where $n \geq m+r$, and let $T_{x} X=H \subset k^{n}$ be a linear subspace given by a system of independent linear equations $B_{l}=\sum b_{l k} x_{k}, l=1, \ldots, r$. By abuse of notation we denote by $A$ the matrix (in the canonical bases in $k^{n}$ and $k^{m}$ ) of the mapping $A$. Let $C=$ be an $(m+r) \times n$ matrix given by rows $A_{1}, \ldots, A_{m} ; B_{1}, \ldots, B_{r}$ (we identify $A_{i}=\sum a_{i k} x_{k}$ with the vector $\left(a_{j 1}, \ldots, a_{j n}\right)$, similarly for $\left.B_{l}\right)$.

For an index $I=\left(i_{1}, \ldots, i_{m+r}\right) \subset\{1, \ldots, n\}$ let $M_{I}(x)$ denote the $((m+r) \times(m+r))$ minor of $C$ given by columns indexed by $I$. For integers $j \in I, 1 \leq k \leq m$, we denote by $M_{I(k, j)}(x)$ the $(m+r-1) \times(m+r-1)$ minor obtained by deleting the $j$ th column and the $k$ th row. Note that we delete only $A_{k}, 1 \leq k \leq m$, rows!

Hence $M_{I}$ and $M_{I(k, j)}$ are regular (restriction of polynomials) functions on $X$. We now define a family of rational functions on $X$ :

$$
W_{I(k, j)}(x)=M_{I}(x) / M_{I(k, j)}(x),
$$

where for $M_{I(k, j)} \equiv 0$, we put $W_{I(k, j)} \equiv 0$. We write $b=\left(b_{1}, \ldots, b_{r}\right)$ and $(f, b): \mathbb{C}^{n} \rightarrow$ $\mathbb{C}^{m} \times \mathbb{C}^{r}$, here we consider $f_{1}, \ldots, f_{m}$, and $b_{1}, \ldots, b_{r}$ as polynomials on $\mathbb{C}^{n}$.

Let $s=\left(\begin{array}{c}n \\ m+r\end{array}\right)$ and let $M_{I_{1}}, \ldots, M_{I_{s}}$ be all possible main minors of a matrix of $d_{x}(f, b)$. For every index $I_{l}$ take a pair $\left(k_{l}, j_{l}\right)$ which determines an $(m+r-1) \times(m+r-1)$ minor of $M_{I_{l}}$. We denote a sequence $\left(k_{1}, j_{1}\right), \ldots,\left(k_{s}, j_{s}\right)$ by $(k, j) \in \mathbb{N}^{s} \times \mathbb{N}^{s}$ and we consider a rational function:

$$
\Phi_{(k, j)}=\Phi\left(\left(k_{1}, j_{1}\right), \ldots,\left(k_{s}, j_{s}\right)\right): X \rightarrow \mathbb{C}^{m} \times \mathbb{C}^{N},
$$


where the first component of $\Phi_{(k, j)}$ is $f$ and next components are $W_{I_{i}\left(k_{i}, j_{i}\right)}, i=1, \ldots, s$, and all products $x_{l} W_{I_{i}\left(k_{i}, j_{i}\right)}, i=1, \ldots, s ; l=1, \ldots, m$.

We can assume that for some choice of $l$ we have $W_{I_{l}\left(k_{l}, j_{l}\right)} \not \equiv 0$, and consequently dim $\operatorname{cl}\left(\Phi_{(k, j)}(X)\right)=\operatorname{dim} X=n-r$. Here $c l(Y)$ stands for the closure of $Y$ in the strong (or, which is the same, in the Zariski topology). Let $\Gamma(k, j)=\operatorname{cl}\left(\Phi_{(k, j)}(X)\right)$.

Let us recall that $y \in K_{\infty}(f)$ if there exists a sequence $x \rightarrow \infty$ such that

$$
f(x) \rightarrow y \text { and }|x| g^{\prime}(x) \rightarrow 0,
$$

where $g^{\prime}(x)=g^{\prime}\left(d_{x} f, T_{x} X\right)$. We have

Lemma 4.3.

$$
K(f)=K_{0}(f) \cup K_{\infty}(f)=\mathbb{C}^{m} \cap \bigcup_{(k, j)} \Gamma(k, j),
$$

where we identify $\mathbb{C}^{m}$ with $\mathbb{C}^{m} \times(0, \ldots, 0)$.

Proof. Indeed, if $y \in K_{0}(f)$ then there is a critical point $x_{0} \in X$, such that $y=f\left(x_{0}\right)$. Since $d_{x_{0}} f$ is singular on $T_{x_{0}} X$, we have $g^{\prime}\left(x_{0}\right)=0$, consequently for any sequence $x \rightarrow x_{0}$ we have $g^{\prime}(x) \rightarrow 0$. In particular for every index $I_{i}$ there are integers $\left(k_{i}, j_{i}\right)$ such that $M_{I_{i}} / M_{I_{r}\left(k_{i}, j_{i}\right)}(x) \rightarrow 0$. This means that $y \in \Gamma(k, j) \cap \mathbb{C}^{m}$ where $(k, j)=$ $\left(\left(k_{1}, j_{1}\right), \ldots,\left(k_{s}, j_{s}\right)\right)$.

Similarly, if $y \in K_{\infty}(f)$, then there is a sequence $x \rightarrow \infty$, such that for every $I_{i}$ there are integers $\left(k_{i}, j_{i}\right)$, such that $|x| M_{I_{i}} / M_{I_{r}\left(k_{i}, j_{i}\right)}(x) \rightarrow 0$. This also gives $y \in \Gamma(k, j) \cap \mathbb{C}^{m}$ with $(k, j)=\left(\left(k_{1}, j_{1}\right), \ldots,\left(k_{s}, j_{s}\right)\right)$.

Conversely, if $y \in \Gamma(k, j) \cap \mathbb{C}^{m}$, then we can choose a sequence $x \rightarrow a$, where $a \in \mathbb{C}^{n}$ or $a=\infty$, such that $M_{I_{r}} / M_{I_{r}\left(k_{r}, j_{r}\right)}(x) \rightarrow 0$ and $|x| M_{I_{r}} / M_{I_{r}\left(k_{r}, j_{r}\right)}\left(x_{n}\right) \rightarrow 0$. If $a \in \mathbb{C}^{n}$, this implies that all $M_{I}(a)=0$, i.e., $a$ is a critical point of $f$, hence $y=f(a) \in K_{0}(f)$. Otherwise we have that $\mid x \| g^{\prime}(x) \rightarrow 0$ and $f(x) \rightarrow y$, i.e., $y \in K_{\infty}(f)$. By Theorem 3.3 we have that $K(f) \neq \mathbb{C}^{m}$, hence $\mathbb{C}^{m} \cap \bigcup_{(k, j)} \Gamma(k, j) \neq \mathbb{C}^{m}$.

In particular we have proved that the set $K(f)$ is algebraic. For an index $J=$ $\left(i_{1}, \ldots, i_{m}\right) \subset\{1, \ldots, n\}$ and numbers $k \in I, j \in\{1, \ldots, m\}$, let $\alpha_{J(k, j)}$ denote a complex number. For every index $I$ take

$$
W_{I}(x)=M_{I} /\left(\sum_{J, k, j} \alpha_{J(k, j)} M_{J(k, j)}\right),
$$

where $M_{J(k, j)}$ denotes the $(m-1) \times(m-1)$ minor which is created from $M_{J}$ by deleting the $j$ th row and $k$ th column (recall that we delete only $A_{j}$ rows!).

Now consider the rational mapping $\Phi: X \ni x-\rightarrow\left(f(x), W_{I_{1}}(x), x_{1} W_{I_{1}}(x), \ldots\right.$, $\left.x_{n} W_{I_{1}}(x), \ldots, W_{I_{s}}(x), x_{1} W_{I_{s}}(x), \ldots, x_{n} W_{I_{s}}(x)\right) \in \mathbb{C}^{m} \times \mathbb{C}^{N}$. Let $\Gamma=\overline{\Phi(X)}$.

Lemma 4.4. For sufficiently general numbers $\alpha_{J(k, j)}$ we have

$$
K(f)=K_{0}(f) \cup K_{\infty}(f)=\Gamma \cap \mathbb{C}^{m},
$$

where we identify $\mathbb{C}^{m}$ with $\mathbb{C}^{m} \times(0, \ldots, 0)$. 
Proof. We take a dense countable subset $E=\left\{y_{1}, y_{2}, y_{3}, \ldots\right\}$ of $K(f)$. By our previous consideration and by Proposition 2.5 for every $y_{k}$ there is a sequence $x_{k j} \in X$ and $x_{k j} \rightarrow a_{k}$, where $a_{k} \in X$ or $a_{k}=\infty$, such that

(a) $f\left(x_{k j}\right) \rightarrow y_{k}$,

(b) if $\left|M_{J_{k j}\left(a_{k j}, b_{k j}\right)}\left(x_{k j}\right)\right|$ denotes appropriate maximal minors, then for every index $I$ we have: $\left|M_{I}\left(x_{k j}\right)\right| /\left|M_{J_{k j}\left(a_{k j}, b_{k j}\right)}\left(x_{k j}\right)\right| \rightarrow 0$ and $\left|x_{k j}\right|\left|M_{I}\left(x_{k j}\right)\right| /\left|M_{J_{k j}\left(a_{k j}, b_{k j}\right)}\left(x_{k j}\right)\right|$ $\rightarrow 0$ (the first limit is important only if $a_{k} \in \mathbb{C}^{n}$ ).

We fix $k$. We assume that $a_{k}=\infty$, the other case can be done similarly. By the Dirichlet box principle we can assume that there exist $J, a, b$ such that $\left|M_{J(a, b)}\right|\left(x_{k j}\right) \mid$ are maximal among others minors of this type (if it is necessary we take a subsequence).

In particular we can assume that all ratios $\left|M_{J^{\prime}(c, d)}\left(x_{k j}\right)\right| /\left|M_{J(a, b)}\left(x_{k j}\right)\right|$ are (defined and) bounded by 1 . Thus (again taking a subsequence) we can assume that all these limits exist. Let $\gamma\left(k, J^{\prime}, c, d\right)=\lim _{j \rightarrow \infty} M_{J^{\prime}(c, d)}\left(x_{k j}\right) / M_{J(a, b)}\left(x_{k j}\right)$.

Since a countable family of hyperplanes cannot fill the whole of the affine space, we can find numbers $A_{J^{\prime}, c, d}$ such that for all $k=1,2, \ldots$ we have

$$
\sum_{J^{\prime}, c, d} A_{J^{\prime}, c, d} \gamma\left(k, J^{\prime}, c, d\right)=\varepsilon_{k} \neq 0
$$

Now take $\alpha_{I(c, d)}=A_{I, c, d}$ as our general coefficients. Note that $E \subset \Gamma$. Indeed for $j$ large enough we have

$$
\left|\sum \alpha_{I(c, d)} M_{I(c, d)}\right| \geq\left|M_{J(a, b)}\left(x_{k j}\right)\right|\left|\varepsilon_{k} / 2\right|
$$

and consequently for every index $I$,

$$
\left|x_{k j}\right|\left|M_{I}\left(x_{k j}\right)\right| /\left(\left|\sum \alpha_{J^{\prime}(k, j)} M_{J^{\prime}(k, j)}\left(x_{k j}\right)\right|\right) \leq\left|2 / \varepsilon_{k}\right|\left|x_{k j}\right|\left|M_{I}\left(x_{k j}\right)\right| /\left|M_{J(a, b)}\left(x_{k j}\right)\right| \rightarrow 0 .
$$

Thus we have $\lim \Phi\left(x_{k j}\right)=y_{k}$, hence $E \subset \Gamma \cap \mathbb{C}^{m}$. Since $\Gamma \cap \mathbb{C}^{m}$ is a closed set, we obtain $K(f) \subset \Gamma \cap \mathbb{C}^{m}$.

Now take $y \in \Gamma \cap \mathbb{C}^{m}$, then there is a sequence $x_{j} \rightarrow a$, where $a \in C^{n}$ or $a=\infty$, such that $y=\lim f\left(x_{j}\right)$ and

$$
\left|x_{j}\right|\left|M_{I}\left(x_{j}\right)\right| /\left|\sum \alpha_{J^{\prime}(k, j)} M_{J^{\prime}(k, j)}\left(x_{j}\right)\right| \rightarrow 0 .
$$

As before we can assume that $a=\infty$ and that the minor $\left|M_{J}(a, b)\left(x_{j}\right)\right|$ is maximal (and non-zero) for every $j$ and that all limits

$$
\gamma\left(J^{\prime}, c, d\right)=\lim _{j \rightarrow \infty} M_{J^{\prime}(c, d)}\left(x_{j}\right) / M_{J(a, b)}\left(x_{j}\right)
$$

exist. Let

$$
\varepsilon=\sum_{J^{\prime}, c, d} A_{J^{\prime}, c, d} \gamma\left(J^{\prime}, c, d\right)
$$

If $\varepsilon \neq 0$, then

$$
\left|x_{j}\right|\left|M_{I}\left(x_{j}\right)\right| /\left|\sum \alpha_{J^{\prime}(k, j)} M_{J^{\prime}(k, j)}\left(x_{j}\right)\right| \geq|1 /(2 \varepsilon)|\left|x_{j}\right|\left|M_{I}\left(x_{j}\right)\right| /\left|M_{J(a, b)}\left(x_{j}\right)\right|
$$


and since the first term tends to zero, we also have $\left|x_{j}\right|\left|M_{I}\left(x_{j}\right)\right| /\left|M_{J(a, b)}\left(x_{j}\right)\right| \rightarrow 0$, which means that $y \in K(f)$. If $\varepsilon=0$ we can modify sequence $x_{j}$ in this way such that $\varepsilon_{j}:=\sum_{J^{\prime}, c, d} A_{J^{\prime}, c, d} M\left(J^{\prime}, c, d\right) / M(J, a, b) \neq 0$ (note that by the construction the function $\left.\sum_{J^{\prime}, c, d} A_{J^{\prime}, c, d} M\left(J^{\prime}, c, d\right)(x) \not \equiv 0\right)$. Of course $\lim \varepsilon_{j}=\varepsilon=0$. We have

$$
\left|\varepsilon_{j}\right|\left|x_{j}\right|\left|M_{I}\left(x_{j}\right)\right| /\left|\sum \alpha_{J^{\prime}(k, j)} M_{J^{\prime}(k, j)}\left(x_{j}\right)\right| \geq\left|x_{j}\right|\left|M_{I}\left(x_{j}\right)\right| /\left|M_{J(a, b)}\left(x_{j}\right)\right|
$$

and again we have $\left|x_{j}\right|\left|M_{I}\left(x_{j}\right)\right| /\left|M_{J(a, b)}\left(x_{j}\right)\right| \rightarrow 0$. Hence $y \in K(f)$ and $\Gamma \cap C^{m} \subset$ $K(f)$. Finally we have $K(f)=\Gamma \cap \mathbb{C}^{m}$.

Proof of Theorem 4.1. It is enough to estimate a degree of a hypersurface in which the set $\Gamma \cap \mathbb{C}^{m}$ is contained. Let $M \subset \mathbb{C}^{m} \times \mathbb{C}^{N}$ be a linear subspace of dimension $n-r+1$, which contains the subspace $L=\mathbb{C}^{m}=\mathbb{C}^{m} \times(0, \ldots, 0)$. By Lemma 4.1 there is a projection $p: \Gamma \rightarrow M$ such that $p(\Gamma) \cap \mathbb{C}^{m} \neq \mathbb{C}^{m}$. Hence it is enough to estimate the degree of a hypersurface $p(\Gamma) \subset M$. To do this we use Lemma 4.2. In fact we have to estimate the degree of the image of the rational function $p \circ \Phi: X-\rightarrow M \cong \mathbb{C}^{n-r+1}$. In particular $p \circ \Phi=\left(P_{1} / Q, \ldots, P_{n-r+1} / Q\right)$, where

$$
\operatorname{deg} P_{i} \leq d+((m-1)(d-1)+r(D-1))
$$

and

$$
\operatorname{deg} Q \leq(m-1)(d-1)+r(D-1) .
$$

Finally by Lemma 4.2 we have

$$
\operatorname{deg} p(\Gamma) \leq(d+(m-1)(d-1)+r(D-1))^{n-r} \operatorname{deg} X,
$$

which proves the first estimate in Theorem 4.1. To get the second one it is enough to apply the Bezout theorem.

We immediately deduce an analogous statement in the real case:

Corollary 4.1. Let $X \subset \mathbb{R}^{n}$ be a smooth affine variety of dimension $n-r \geq m$. Assume that $I(X)=\left\{b_{1}, \ldots, b_{w}\right\}$, where $\operatorname{deg} b_{i} \leq D$. Let $f=\left(f_{1}, \ldots, f_{m}\right): X \rightarrow \mathbb{R}^{m}$ be a polynomial dominant mapping and let $\operatorname{deg} f_{i} \leq d$. The set $K(f)$ is a closed semialgebraic subset of $\mathbb{R}^{m}$ and it is contained in a real hypersurface of degree at most $(d+(m-1)(d-1)+(D-1) r)^{n-r} D^{r}$.

In a particularly interesting case where $f$ is a polynomial function on a hypersurface we have:

Corollary 4.2. If $X$ is a smooth hypersurface of degree $D$ in $\mathbb{C}^{n}$ and $f: X \rightarrow \mathbb{C}$ is a polynomial of degree $d$, then the set $K(f)$ has at most $(d+D-1)^{n-1} D$ points. In particular $f$ may have at most $(d+D-1)^{n-1} D$ atypical fibers.

The following example shows that the estimate in Corollary 4.2 is asymptotically sharp. 
Example 4.1. Let $X \subset \mathbb{P}^{n}$ be a smooth projective hypersurface of degree $D$. It is well known that the degree of the dual hypersurface to $X$ is $D(D-1)^{n-1}$, see, e.g., [4]. This means in particular that there is a projective subspace $W \subset \mathbb{P}^{n}$ of codimension 2, such that there is exactly $D(D-1)^{n-1}$ tangent hyperplanes to $X$ which contain $W$. We take an affine system of coordinates in $\mathbb{P}^{n}$ in such a way that $W$ is contained in the hyperplane at infinity, and all these tangent spaces which contain $W$ are different from the hyperplane at infinity. Let $f$ be a homogeneous linear function which describes the subspace $W$. Then $f$ considered as a polynomial on the affine part of $X$ has at least $D(D-1)^{n-1}$ generalized critical values, because it has at least $D(D-1)^{n-1}$ singular fibers. This means that our estimation is nearly sharp.

Clearly Corollary 4.1 yields in the real case:

Corollary 4.3. If $X$ is a smooth hypersurface of degree $D$ in $\mathbb{R}^{n}$ and $f: X \rightarrow \mathbb{R}$ is a polynomial of degree $d$, then the set $K(f)$ has at most $(d+D-1)^{n-1} D$ points. In particular $f$ has at most $(d+D-1)^{n-1} D$ non-generic fibers and consequently there is at most $(d+D-1)^{n-1} D+1$ types of generic fibers of $f$.

Note that from the proof of Theorem 4.1 it follows that to give an estimation of the degree of $K(f)$ it is not necessary to know the system of generators of $I(X)$ (that in general is difficult). It is enough to find polynomials $\left\{h_{1}, \ldots, h_{r}\right\} \subset I(X), r=$ codim $X$, such that $\operatorname{Jac}\left(h_{1}, \ldots, h_{r}\right)$ does not vanish identically on $X$ and then we can put $D=\max \left\{\operatorname{deg} h_{i}\right\}$. Below we show that in this way we can always take $D=\operatorname{deg} X$.

Theorem 4.2. Let $X \subset \mathbb{C}^{n}$ be a smooth affine variety of dimension $n-r \geq m$. Let $f=\left(f_{1}, \ldots, f_{m}\right): X \rightarrow \mathbb{C}^{m}$ be a polynomial dominant mapping and let $\operatorname{deg} f_{i} \leq d$. Assume that $\operatorname{deg} X=D$. Then the set $K(f)$ is a proper algebraic subset of $\mathbb{C}^{m}$ and is contained in a hypersurface of degree at most

$$
(d+(m-1)(d-1)+(D-1) r)^{n-r} D .
$$

Proof. It is enough to construct polynomials $h_{1}, \ldots, h_{r}$ of degree $D=\operatorname{deg} X$, which vanish on $X$ and for which $\operatorname{Jac}\left(h_{1}, \ldots, h_{r}\right)$ does not vanish identically on $X$.

Let us take a point $x \in X$. Let $S$ be the closure of the union of all secants $x y$, where $y \in X$ is another point of $X$. It is easy to see that $\operatorname{dim} S \leq n-r+1$ and the projective closure $\bar{S}$ of $S$ contains the projective closure of $X$.

Now on the hyperplane at infinity, $H_{\infty}$, we choose a system of homogeneous coordinates $x_{1}, \ldots, x_{n-r}, x_{n-r+1}, \ldots, x_{n}$ in such a way that for every $j>n-r$ we have $\left\{x_{1}=0, \ldots, x_{n-r}=0, x_{j}=0\right\} \cap \bar{S}=\emptyset$. Of course every sufficiently general system of coordinates has this property. We can extend the coordinate system on $H_{\infty}$ in an obvious way to a coordinate system on the whole of $\mathbb{P}^{n}$ (by adding a new variable $x_{0}$ ).

Now for every $j>n-r$ we consider the projection $\pi_{j}: X \ni x \rightarrow\left(x_{1}, \ldots, x_{n-r}, x_{j}\right) \in$ $\mathbb{C}^{n-r+1}$. By the construction the mapping $\pi_{j}$ is proper and birational (the last property follows from the fact that $\left(\pi_{j}\right)^{-1}\left(\pi_{j}(x)\right)=\{x\}$ and that $\pi_{j}$ is smooth at $\left.x\right)$. The image $X_{j}:=\pi_{j}(X)$ is a hypersurface in $\mathbb{C}^{n-r+1}$. Let $h_{s}$ be a reduced equation of $X_{n-r+s}$. Then $h_{s}$ vanishes on $X$ and $\partial h_{s} / \partial x_{n-r+s}$ does not vanish identically on $X$. Now it is 
easy to check that polynomials $h_{1}, \ldots, h_{r}$ (of degree $D=\operatorname{deg} X$ ) vanish on $X$ and $\operatorname{Jac}\left(h_{1}, \ldots, h_{r}\right)$ does not vanish identically on $X$.

Corollary 4.4. Let $X$ be a smooth algebraic variety of dimension $k$ and degree $D$ in $\mathbb{C}^{n}$ and let $f: X \rightarrow \mathbb{C}$ be a polynomial of degree $d$. Then the set $K(f)$ has at most $(d+(D-1)(n-k))^{k} D$ points. In particular $f$ may have at most $(d+(D-1)(n-k))^{k} D$ atypical fibers.

Corollary 4.5. Let $X$ be a smooth algebraic variety of dimension $k$ and degree $D$ in $\mathbb{R}^{n}$ and let $f: X \rightarrow \mathbb{R}$ be a polynomial of degree $d$. Then the set $K(f)$ has at most $(d+(D-1)(n-k))^{k} D$ points. In particular $f$ may have at most $(d+(D-1)(n-k))^{k} D$ atypical fibers and consequently there is at most $(d+(D-1)(n-k))^{k} D+1$ types of generic fibers of $f$.

We can also apply our results to rational functions $f: \mathbb{C}^{n} \rightarrow \mathbb{C}^{m}$. For simplicity we formulate the result for a rational function $f: \mathbb{C}^{n} \rightarrow \mathbb{C}$ (we leave it to the reader to formulate all obvious definitions). The general case can be done similarly.

Corollary 4.6. If $f=P / Q: \mathbb{C}^{n} \rightarrow \mathbb{C}$ is a rational function of degree $d$ (i.e., max $\operatorname{deg} P, \operatorname{deg} Q=d)$, then the set $K(f)$ has at most $(2 d+1)^{n}(d+1)$ points. In particular $f$ may have at most $(2 d+1)^{n}(d+1)$ atypical fibers.

Proof. We consider the hypersurface $X=\left\{(x, z) \in \mathbb{C}^{n} \times \mathbb{C}: z Q(x)=1\right\}$. Thus the function $f$ can be considered as the polynomial function $F=z P(x)$ on the hypersurface $X$. Since this hypersurface and the function $F$ have degrees at most $d+1$, we have that the set $K(f)$ has at most $(2 d+1)^{n}(d+1)$ points.

Now we consider the special case $n=m$. Recall that a mapping $f: X \rightarrow k^{n}$ is not proper at a point $y \in k^{m}$ if there is no neighborhood $U$ of $y$ such that $f^{-1}(\bar{U})$ is compact. In other words, $f$ is not proper at $y$ if there is a sequence $x_{l} \rightarrow \infty$ such that $f\left(x_{l}\right) \rightarrow y$. Let $S_{f}$ denote the set of points at which the mapping $f$ is not proper. It is well known (see [5] and [6]) that in the case $k=\mathbb{C}$ the set $S_{f}$ is either an empty set or is a hypersurface. Following the proof of Proposition 4.1 in [8] we obtain:

Proposition 4.1. Let $f=\left(f_{1}, \ldots, f_{n}\right): X \rightarrow \mathbb{C}^{n}$ be a dominant polynomial mapping. Then the set $K(f)$ of generalized critical values is either empty (so $f$ is an automorphism) or is a hypersurface. Moreover, $K(f)=K_{0}(f) \cup S_{f}=B(f)$. In particular the bifurcation set $B(f)$ is either empty (so $f$ is an automorphism) or is a hypersurface.

In the real case $K(f)$ need not be a hypersurface, in particular $K_{\infty}(f)$ may be of codimension at least 2 (see [7]). However, arguing as in the proof of Proposition 3.1 in [12] we can easily prove the following. 
Proposition 4.2. Assume that $X$ is smooth and algebraic of dimension $n$ and let $f=\left(f_{1}, \ldots, f_{n}\right): X \rightarrow \mathbb{R}^{n}$ be a polynomial mapping (or more generally $C^{1}$ with a semialgebraic graph). Then $K_{\infty}(f)=S_{f}$ and consequently $B(f)=K_{0}(f) \cup S_{f}$.

\section{Computations}

In this section we use the Gröbner basis to compute the set $K(f)$ effectively. We recall the definition of the Gröbner basis. Assume that in the set of monomials in $\mathbb{C}\left[x_{1}, \ldots, x_{n}\right]$ we have the ordering induced by the lexicographic ordering in $\mathbb{N}^{n}$, i.e., $a_{\alpha} x^{\alpha}>a_{\beta} x^{\beta}$, if $\alpha>\beta$ (in this paper we consider only this ordering). By inP $=a_{d} x^{d}$ we denote the initial form of a polynomial $P=\sum a_{\alpha} x_{1}^{\alpha_{1}} \cdots x_{n}^{\alpha_{n}} \in \mathbb{C}\left[x_{1}, \ldots, x_{n}\right]$, where $d=\max \{\alpha=$ $\left.\left(\alpha_{1}, \ldots, \alpha_{n}\right) ; a_{d} \neq 0\right\}$. We have the following basic definition (see [15]):

Definition 5.1. A finite subset $\mathcal{B} \subset I \subset \mathbb{C}\left[x_{1}, \ldots, x_{n}\right]$ of an ideal $I$ is called a Gröbner basis of this ideal if the set $\{$ in $P ; P \in \mathcal{B}\}$ generates the ideal generated by all initial forms of the ideal I.

The Gröbner basis of the ideal $I$ is a basis of this ideal, moreover it can be easily computed by arithmetical operations only. We have the following basic fact (see [15]):

Theorem 5.1. Consider the ring $\mathbb{C}\left[x_{1}, \ldots, x_{n} ; y_{1}, \ldots, y_{m}\right]$. Let $V \subset \mathbb{C}^{n} \times \mathbb{C}^{m}$ be an algebraic set and let $p: \mathbb{C}^{n} \times \mathbb{C}^{m} \rightarrow \mathbb{C}^{m}$ denote the projection. Assume that $\mathcal{B}$ is a Gröbner basis of the ideal $I(V)$. Then $\mathcal{B} \cap \mathbb{C}\left[y_{1}, \ldots, y_{m}\right]$ is a Gröbner basis of the ideal $I(p(V))=I(c l(p(V)))$.

Proof. Observe that $I(p(V))=I(V) \cap \mathbb{C}\left[y_{1}, \ldots, y_{m}\right]$ and then use Proposition 4.3 of [15].

\subsection{A Sketch of an Algorithm}

Let $X$ be a smooth affine variety of dimension $n-r$ and let $I(X)=\left\{b_{1}, \ldots, b_{w}\right\}$. Let $f=\left(f_{1}, \ldots, f_{m}\right): X \rightarrow \mathbb{C}^{m}$ be a polynomial dominant mapping. Then the set $K(f)$ can be computed as follows.

We can choose polynomials $b_{1}, \ldots, b_{r} \in I(X)$ such that rank $\left\{\operatorname{grad} b_{1}, \ldots, \operatorname{grad} b_{r}\right\}=$ $r$ on some non-empty open subset of $X$. We consider the rational mapping:

$$
\begin{aligned}
\Phi\left(\left(k_{1}, j_{1}\right), \ldots,\left(k_{s}, j_{s}\right)\right): X \ni x- \\
\rightarrow\left(f(x), W_{I_{1}\left(k_{1}, j_{1}\right)}(x), x_{1} W_{I_{1}\left(k_{1}, j_{1}\right)}(x),\right. \\
\left.\quad \ldots, x_{n} W_{I_{1}\left(k_{1}, j_{1}\right)}(x), \ldots, W_{I_{s}\left(k_{s}, j_{s}\right)}(x), x_{1} W_{I_{s}\left(k_{s}, j_{s}\right)}(x), \ldots, x_{n} W_{I_{s}\left(k_{s}, j_{s}\right)}(x)\right) \\
\quad \mathbb{C}^{m} \times \mathbb{C}^{N}
\end{aligned}
$$

which is constructed exactly as in the proof of Theorem 4.1. Recall that

$$
\Gamma\left(\left(k_{1}, j_{1}\right), \ldots,\left(k_{s}, j_{s}\right)\right)=\operatorname{cl}\left(\Phi\left(\left(k_{1}, j_{1}\right), \ldots,\left(k_{s}, j_{s}\right)\right)\left(\mathbb{C}^{n}\right)\right) .
$$


We know also that

$$
K(f)=L \cap\left(\bigcup_{\left(\left(k_{1}, j_{1}\right), \ldots,\left(k_{s}, j_{s}\right)\right)} \Gamma\left(\left(k_{1}, j_{1}\right), \ldots,\left(k_{s}, j_{s}\right)\right)\right),
$$

where $L=\mathbb{C}^{m} \times(0, \ldots, 0)$. First we compute the ideal of the set $\Gamma\left(\left(k_{1}, j_{1}\right), \ldots,\left(k_{s}, j_{s}\right)\right)$.

To this end we restrict the mapping $\Phi(k, j)$ to an open dense subset $U$ on which this mapping is regular. In particular we can choose the set $U=X \backslash \bigcup_{r=1}^{s}\left\{M_{I_{r}\left(k_{r}, j_{r}\right)}=0\right\}$. The set $U$ can be identified with the set

$$
\begin{aligned}
V\left(\left(k_{1}, j_{1}\right), \ldots,\left(k_{s}, j_{s}\right)\right) & \\
:= & \left\{\left(x, z_{1}, \ldots, z_{s}\right) \in \mathbb{C}^{n} \times \mathbb{C}^{s}: b_{j}=0, j=1, \ldots, w ;\right. \\
& \left.M_{I_{r}\left(k_{r}, j_{r}\right)} z_{r}=1 ; r=1, \ldots, s\right\} .
\end{aligned}
$$

Now we can consider a morphism

$$
\Psi\left(\left(k_{1}, j_{1}\right), \ldots,\left(k_{s}, j_{s}\right)\right): V\left(\left(k_{1}, j_{1}\right), \ldots,\left(k_{s}, j_{s}\right)\right) \rightarrow \mathbb{C}^{m} \times \mathbb{C}^{N}
$$

defined by

$$
\begin{aligned}
(x, z) \rightarrow( & f(x), z_{1} M_{I_{1}\left(k_{1}, j_{1}\right)}(x), x_{1} z_{1} M_{I_{1}\left(k_{1}, j_{1}\right)}(x), \ldots, x_{n} z_{1} M_{I_{1}\left(k_{1}, j_{1}\right)}(x), \\
& \left.\ldots, z_{s} M_{I_{s}\left(k_{s}, j_{s}\right)}(x), x_{1} z_{s} M_{I_{s}\left(k_{s}, j_{s}\right)}(x), \ldots, x_{n} z_{s} M_{I_{s}\left(k_{s}, j_{s}\right)}(x)\right) .
\end{aligned}
$$

Denote $\Psi\left(\left(k_{1}, j_{1}\right), \ldots,\left(k_{s}, j_{s}\right)\right):=\left(\psi_{1}(x, z), \ldots, \psi_{m+N}(x, z)\right)$. It is easy to see that

$$
\Gamma\left(\left(k_{1}, j_{1}\right), \ldots,\left(k_{s}, j_{s}\right)\right)
$$

is the closure of

$$
\Psi\left(\left(k_{1}, j_{1}\right), \ldots,\left(k_{s}, j_{s}\right)\right)\left(V\left(\left(k_{1}, j_{1}\right), \ldots,\left(k_{s}, j_{s}\right)\right)\right) .
$$

Let $G\left(\left(k_{1}, j_{1}\right), \ldots,\left(k_{s}, j_{s}\right)\right)=\operatorname{graph}\left(\Psi\left(\left(k_{1}, j_{1}\right), \ldots,\left(k_{s}, j_{s}\right)\right)\right)$. A basis of the ideal $I$ of the set $G\left(\left(k_{1}, j_{1}\right), \ldots,\left(k_{s}, j_{s}\right)\right)$ in the ring $\mathbb{C}[x, z, y]$ is given by the polynomials

$$
\left\{z_{r} M_{I_{r}\left(k_{r}, j_{r}\right)}(x)-1\right\}_{r=1, \ldots, s} \cup\left\{y_{i}-\psi_{i}(x, z)\right\}_{i=1, \ldots, m+N} .
$$

Thus by Theorem 5.1 to compute a basis $\mathcal{B}\left(\left(k_{1}, j_{1}\right), \ldots,\left(k_{s}, j_{s}\right)\right)$ of the ideal of the set $\operatorname{cl}\left(\Gamma\left(\left(k_{1}, j_{1}\right), \ldots,\left(k_{s}, j_{s}\right)\right)\right)$, it is enough to compute a Gröbner basis $\mathcal{A}\left(\left(k_{1}, j_{1}\right), \ldots,\left(k_{s}, j_{s}\right)\right)$ of the ideal $I$ in $\mathbb{C}\left[x_{1}, \ldots, x_{n}, z_{1}, \ldots, z_{s} ; y_{1}, \ldots, y_{m+N}\right]$ and then to take

$$
\mathcal{B}\left(\left(k_{1}, j_{1}\right), \ldots,\left(k_{s}, j_{s}\right)\right)=\mathcal{A}\left(\left(k_{1}, j_{1}\right), \ldots,\left(k_{s}, j_{s}\right)\right) \cap \mathbb{C}\left[y_{1}, \ldots, y_{m+N}\right] .
$$

Consequently, $K(f)=\bigcup_{\left(\left(k_{1}, j_{1}\right), \ldots,\left(k_{s}, j_{s}\right)\right)}\left\{y \in \mathbb{C}^{m}: h(y, 0, \ldots, 0)=0\right.$ for every $h \in$ $\left.\mathcal{B}\left(\left(k_{1}, j_{1}\right), \ldots,\left(k_{s}, j_{s}\right)\right)\right\}$.

Corollary 5.1. Let $X$ be a smooth affine variety and let $I(X)=\left\{b_{1}, \ldots, b_{w}\right\}$. Let $f=\left(f_{1}, \ldots, f_{m}\right): X \rightarrow \mathbb{C}^{m}$ be a dominant polynomial mapping. Let $\sigma$ be a subfield of $\mathbb{C}$ generated by all coefficients of polynomials $f_{i}$ and $b_{j}$. Then there exists a finite family $\left\{g_{1}, \ldots, g_{s}\right\}$ of polynomials from $\sigma\left[y_{1}, \ldots, y_{m}\right]$, such that

$$
K(f)=\left\{y \in \mathbb{C}^{m}: g_{i}(y)=0, i=1, \ldots, s\right\} .
$$




\section{Acknowledgements}

The first author thanks the Instiute of Mathematics of Universite de Savoie and the MaxPlanck-Institut für Mathematik in Bonn for warm hospitality, while this work was carried out.

\section{References}

1. R. Benedetti, J.-J. Risler, Real Algebraic and Semi-Algebraic Sets, Actualités Mathématiques, Hermann, Paris, 1990.

2. J. Bochnak, M. Coste, M.-F. Roy, Real Algebraic Geometry, EMG 36, Springer, New York, 1998.

3. H. Federer, Geometric Measure Theory, Grundlehren Math. Wiss. 153, Springer, New York, 1969.

4. W. Fulton, Intersection Theory, Springer, New York, 1998.

5. Z. Jelonek, The set of points at which a polynomial map is not proper, Ann. Polon. Math. 58 (1993), 259-266.

6. Z. Jelonek, Testing sets for properness of polynomial mappings, Math. Ann. 315 (1999), 1-35.

7. Z. Jelonek, Geometry of real polynomial mappings, Math. Z. 239 (2002), 321-333.

8. Z. Jelonek, On the generalized critical values of polynomial mappings, Manuscripta Math. 110 (2003), $145-157$.

9. Z. Jelonek, On Asymptotic Critical Values and the Rabier Theorem, Banach Center Publication 65, Polish Academy of Science, Warsaw, 2004, pp. 125-133.

10. Z. Jelonek, K. Kurdyka, On asymptotic critical values of a complex polynomial, J. Reine Angew. Math. 565 (2003), 1-11.

11. K. Kurdyka, On a subanalytic stratification satisfying a Whitney property with exponent 1 , Real Algebraic Geometry, Proceedings, Rennes, 1991, LNM 1524, Springer, New York, 1992, 316-322.

12. K. Kurdyka, P. Orro, S. Simon, Semialgebraic Sard theorem for generalized critical values, J. Differential Geom. 56 (2000), 67-92.

13. A. Mostowski, M. Stark, Elementy Algebry Wyższej, PWN, Warszawa, 1968 (in polish).

14. A. Parusiński, On the bifurcation set of complex polynomial with isolated singularities at infinity, Compos. Math. 97 (1995), 369-384.

15. F. Pauer, M. Pfeifhofer, The theory of Gröbner basis, Enseign. Math. 34 (1988), 215-232.

16. P. J. Rabier, Ehresmann's fibrations and Palais-Smale conditions for morphisms of Finsler manifolds, Ann. of Math. 146 (1997), 647-691.

17. A. N. Varchenko, Theorems on the topological equisingularity of families of algebraic varieties and families of polynomial mappings, Math. USSR Izv. 6 (1972), 949-1008.

18. J. L. Verdier, Stratifications de Whitney et Thórème de Bertini-Sard, Invent. Math. 36 (1976), 295-312.

19. A. H. Wallace, Linear sections of algebraic varities, Indiana Univ. Math. J. 20 (1971), 1153-1162.

Received January 30, 2004, and in revised form June 17, 2005. Online publication September 30, 2005. 